\title{
Washing of Fabrics
}

\section{Part 3: Theoretical Analysis on Effective Heat Conductivity of Fiber Assembly}

\author{
By Hirohito Matsui and Kazuo Moriyama, Members, TMSJ
}

Katata Research Center, Toyobo Co., Ltd. Otsu, Shiga, Japan

Based on the Journal of the Textile Machinery Society of Japan, Transaction, Vol. 31, No. 1, T18-27 (1978)

\begin{abstract}
The heat conduction under steady state within a fiber assembly was theoretically analysed, and its effective heat conductivity $\lambda_{e}\left(\mathrm{cal} / \mathrm{cm} \cdot{ }^{\circ} \mathrm{C} \cdot \mathrm{sec}\right)$, was calculated as follows:

In case of the right angle arrangement,

$$
\frac{\lambda_{e}}{\lambda_{w}}=\frac{1}{b} \sum_{k=0}^{\infty}(-1)^{k}\left(b^{n}-\beta b^{-n}\right) A_{n} .
$$

In case of the equilateral triangle arrangement, the above equation can be used, or

$$
\frac{\lambda_{e}}{\lambda_{w}}=\frac{1}{b} \sum_{k=0}^{\infty} 2 A_{n}\left\{\left(\frac{\sqrt{a^{2}+b^{2}}}{2}\right)^{n}-\beta\left(\frac{\sqrt{a^{2}+b^{2}}}{2}\right)-n\right\} \sin n \varphi
$$

where,

$$
n=2 k+1
$$

$\beta=$ parmeter derived from the heat conductivities of fibers and medium

$\lambda_{w}=$ heat conductivity of the medium $\left(\mathrm{cal} / \mathrm{cm} \cdot{ }^{\circ} \mathbf{C} \cdot \mathrm{sec}\right)$

$a, b, \varphi=$ parameters depending on the arrangement of fibers in the fiber assembly

$A_{n}=$ coefficients which satisfy the boundary conditions and can be obtained by applying the least square method along the boundary lines

The results calculated by these equations agreed well with both experimental and reported data. ${ }^{[1,2]}$
\end{abstract}

\section{Introduction}

In the analysis on washing or chemical-adding process of textiles, the material transfer in the complex composed of fibers and water among them must be considered. When this material transfer is assumed to follow the same equation as that in heat conduction, a parameter must be introduced to represent the mobility of chemicals which coresponds to heat conductivity.

Such a parameter can be obtained by the same way as the total effective heat conductivity of the complex could be obtained from each heat conductivity of the fiber and the water.

Reports on such effective heat conductivity of the complex are few. Kamata ${ }^{[1]}$ devided such a complex into very small parts and replaced it by the network model in which many element-points were connected. He then calculated the heat balance at each element-point. In this method, simultaneous linear equations which had more than 200 unknowns were solved using a large capacity computer. So its application was limited in the case that fibers were arranged at right angles against the heat stream. The calculation was also only on large heat conductivity ratio (heat conductivity of fiber/that of water) of $0.1-100$.
Horikawa and co-workers ${ }^{[2]}$ noticed the similarity between electric and heat conductivities, and analyzed theoretically the phenomena that fibers contacted each other and changed their shapes. However, the experiments were performed only in the case in which some medium existed among fibers and the heat conductivity ratio was between $1 / 50$ and 50. In washing, the transfer rate of chemicals in fibers is considered to be very low compared with that in water, and the heat conductivity ratio approaches zero. So, these literatures ${ }^{[1]}{ }^{[2]}$ are insufficient to clarify the transfer phenomena.

When the complex consists of fibers having irregular cross sections, the experiments or the numerical analysis must be performed as in these reports. But when circular fibers are arranged as a lattice with a constant pitch, the theoretical general solution about temperature distribution in the complex can be obtained from another view-point. In this report, the theoretical general solutions are obtained in case of fibers being arranged at right angles or in equilateral triangles, and the temperature distribution and the effective heat conductitvity are calculated when the heat conductivity ratio is $0-\infty$. The results ae also compared with experimental values and the previously mentioned data. 


\section{Temperature distribution and effective heat conductivity}

\subsection{Differential equation}

The case in which heat flows across the fiber axes at right angles, is analysed. Notations used are as followings (c.f. Figs. 1 and 2):

$r$; length of a radius vector in polar coordinates the center of which is on a fiber axis, $(\mathrm{cm})$,

$\theta$; argument measured from the coordinate axis along the heat stream, (rad),

$H(r, \theta)$; function representing the temperature distribution in the medium, $\left({ }^{\circ} \mathrm{C}\right)$,

$F(r, \theta)$; function representing the temperature distribution in the fiber, $\left({ }^{\circ} \mathrm{C}\right)$

In eqs. (1) and (2), $H$ is used in stead of $F$.

$R$; radius of the fiber, $(\mathrm{cm})$,

$\lambda_{f}$; heat conductivity of the fiber, $\left(\mathrm{cal} / \mathrm{cm} \cdot{ }^{\circ} \mathrm{C} \cdot \mathrm{sec}\right)$,

$\lambda w$; heat conductivity of the medium, $\left(\mathrm{cal} / \mathrm{cm} \cdot{ }^{\circ} \mathrm{C} \cdot \mathrm{sec}\right)$,

$\lambda e$; effective heat conductivity of the complex, $\left(\mathrm{cal} / \mathrm{cm} \cdot{ }^{\circ} \mathrm{C}\right.$.

sec $), \quad \beta=\left(\lambda_{w}-\lambda_{f}\right) /\left(\lambda_{w}+\lambda_{f}\right)$

$\alpha=\lambda_{f} / \lambda_{w}$, heat conductivity ratio,

$\varepsilon$; fiber volume ratio in the complex $=$ fiber volume/total volume of fibers and the medium,

$\mathrm{Ho}$; mean temperature gradient multiplied by the fiber radius, $\left({ }^{\circ} \mathrm{C}\right)$,

$a$; fiber pitch along heat stream/the fiber diameter,

$b$; fiber pitch perpendicular to heart stream/the fiber diameter,

$n=2 k+1$

Analyses are performed under the following assumptions.

(1) The medium existing among fibers do not move.

(2) In fibers and the medium, there is no heat generation.

So, this belongs to a two dimentional problem, and the function $H(r, \theta)$ representing the temperature distribution in polar coordinates is written as follows as shown in Kodaira's report ${ }^{[3]}$,

$$
\frac{\partial^{2} H}{\partial r^{2}}+\frac{1}{r} \frac{\partial H}{\partial r}+\frac{1}{r^{2}} \frac{\partial^{2} H}{\partial \theta^{2}}=0
$$

If one solution could be obtained after the variables were seperated, it is,

$$
\begin{aligned}
H= & A+B \ln r+\sum_{n=1}^{\infty}\left\{\left(C_{n} r^{n}+D_{n} r^{-n}\right) \sin n \theta\right. \\
& \left.+\left(E_{n} r^{n}+F_{n} r^{-n}\right) \cos n \theta\right\} \ldots \ldots \ldots \ldots \ldots \ldots
\end{aligned}
$$

where $A, B, C_{n}, D_{n} E_{m}$ and $F_{n}$ are the coefficients determined by boundary conditions. The purpose of this report is to determine these coefficients and obtain the effective heat conductivity. The reason why the polar coordinates are used is that the fiber section is circular and the boundary conditions can be introduced easily.

2.2 Determination of coefficients when fibers are perpendicular to heat stream

As shown in Fig. 1, if the origin of the polar coordinates

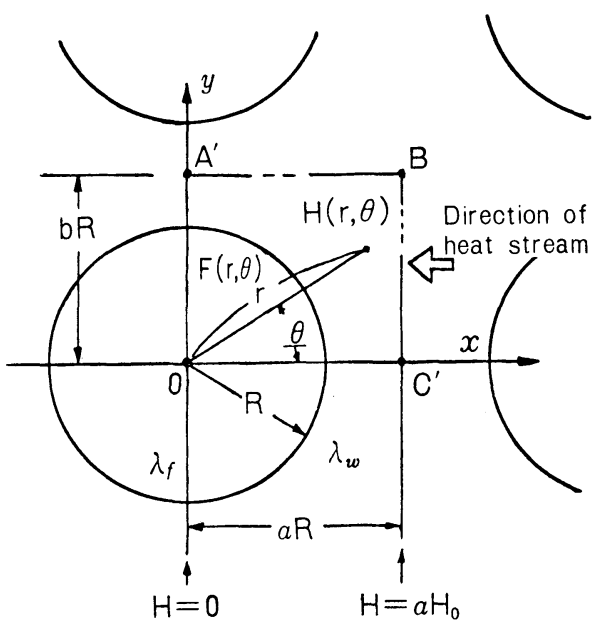

Fig. 1 Coordinates in case of the right angle arrangement

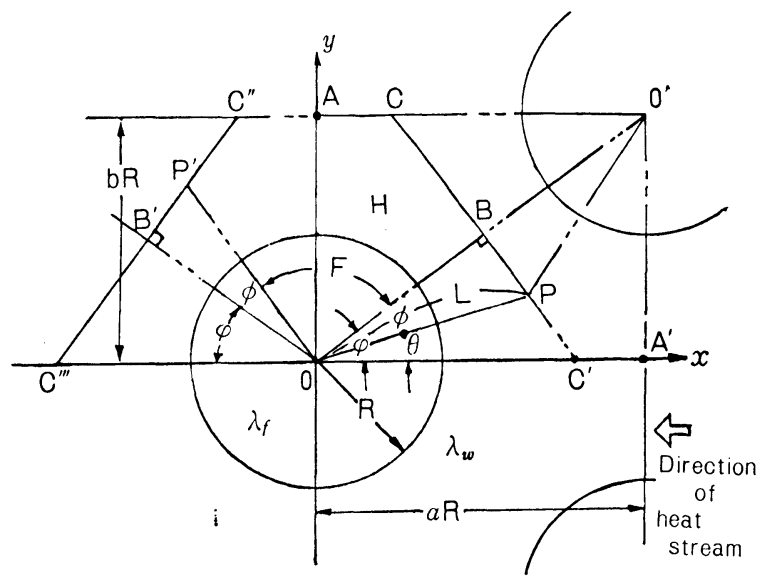

Fig. 2 Coordinates in case of the eqlilateral triangle arrangement

is set on the axis of a fiber, the rectangle $\mathrm{A}^{\prime} O \mathrm{C}^{\prime} \mathrm{B}$ becomes a unit which is repeated in the whole domoain. So, it is sufficient to investigate the temperature distribution only in this rectangle.

Planes $A^{\prime} B$ and $O C^{\prime}$ are adiabatic and planes $A^{\prime} O$ and $B C$ ' are isothermal from the symmetrical property of the temperature distribution. Namely, at $\theta=0$ (in plane $0 C^{\prime}$ ), $\partial H / \partial \theta=0$ and $\partial F / \partial \theta=0$. If the temperature in plane $A^{\prime} 0$ is zero, $H=0$ and $F=0$ at $\theta=\pi / 2$ (in plane $\mathrm{A}^{\prime} 0$ ).

From these two coditions, all of $A, B, C_{n}$ and $D_{n}$ in eq. (2) become zero, and $E_{n}$ and $F_{n}$ are not zero only when $n$ is odd.

Therefor, on defining the coefficients newly as $A_{n}, B_{n}$ and $C_{n}$, we have

$$
H=\sum_{k=0}^{\infty}\left\{A_{n}\left(\frac{r}{R}\right)^{n}+B_{n}\left(\frac{r}{R}\right)^{-n}\right\} \cos n \theta
$$




$$
F=\sum_{k=0}^{\infty} C_{n}\left(\frac{r}{R}\right)^{n} \cos n \theta
$$

Terms of $(r / R)^{-\mathrm{n}}$ do not appear in eq. (4). because $F$ must be finite even at the origin of the coordinates.

On the fiber surface $(r=R)$, the continuity of temperature and heat stream must be realized. So, we have

$$
\begin{aligned}
& (H)_{r=R}=(F)_{r=R} \\
& \lambda_{w}\left(\frac{\partial H}{\partial r}\right)_{r=R}=\lambda_{f}\left(\frac{\partial F}{\partial r}\right)_{r=R}
\end{aligned}
$$

From these and eqs (3) and (4), we have

$$
\left\{\begin{array}{l}
A_{n}+B_{n}=C_{n} \\
\lambda_{w}\left(A_{n}-B_{n}\right)=\lambda_{f} C_{n}
\end{array}\right.
$$

On defining $\beta=\left(\lambda_{w}-\lambda_{f}\right) /\left(\lambda_{w}+\lambda_{f}\right)$, we have

$$
\left.\begin{array}{l}
B_{n}=\beta A_{n} \\
C_{n}=(1+\beta) A_{n}
\end{array}\right\}
$$

Here, the coefficients not yet decided are $A_{n}$ when $n$ is odd. Making $A_{n}$ dimentionless, we have

$$
\begin{aligned}
& H / H_{0}=\sum_{k=0}^{\infty} A_{n}\left\{\left(\frac{r}{R}\right)^{n}+\beta\left(\frac{r}{R}\right)^{-n}\right\} \cos n \theta \cdots \cdots(6) \\
& F / H_{0}=\sum_{k=0}^{\infty} A_{n}(1+\beta)\left(\frac{r}{R}\right)^{n} \cos n \theta \quad \cdots \cdots \cdots \cdots(7)
\end{aligned}
$$

$A_{n}$ must be determined so as to fulfill the boundary conditions not yet used, namely adiabatic on plane $\mathrm{A}^{\prime} \mathrm{B}$, and $H=$ a $H_{0}$ (constant) on plane $\mathrm{BC}$ ':

$$
\begin{gathered}
H=a H_{0} \text { where } x=a R \text { and } 0 \leqq y \leqq b R, \text { and } \\
\frac{\partial H}{\partial y}=\frac{\partial H}{\partial r} \sin \theta+\frac{\partial H}{\partial \theta}-\frac{\cos \theta}{r}=0
\end{gathered}
$$

where $0 \leqq x \leqq a R$ and $y=b R$.

From the above relations and eq. (6), we have

$$
a=\sum_{k=0}^{\infty} A_{n}\left\{\left(\frac{a}{\cos \theta}\right)^{n}+\beta\left(\frac{\cos \theta}{a}\right)^{n}\right\} \cos n \theta \cdots \cdots(8)
$$

where $0 \leqq \theta \leqq \tan ^{-1} b / a$, and

$$
\begin{aligned}
& 0=\sum_{k=0}^{\infty} A_{n} n\left\{\left(\frac{b}{\sin \theta}\right)^{n} \sin (n-1) \theta\right. \\
&\left.+\beta\left(\frac{\sin \theta}{b}\right)^{n} \sin (n+1) \theta\right\} \frac{\sin \theta}{b}
\end{aligned}
$$

where $\tan ^{-1} b / a \leqq \theta \leqq \pi / 2$.

When $A_{n}$ is determined, effective heat conductivity $\lambda_{e}$ can be obtained by considering the heat stream passing through rectangle A'OC'B in Fig. 1. Namely, the temperature in plane $\mathrm{BC}^{\prime}$ is $a \mathrm{Ho}$, and that in plane $\mathrm{A}^{\prime} \mathrm{O}$ is zero, and the distance $\mathrm{OA}^{\prime}$ is $b R$, so the amount of the above mentioned heat stream $q(\mathrm{cal} / \mathrm{sec})$ is

$$
q=\lambda_{e} b R \frac{a H_{0}}{a R}=\lambda_{e} b H_{0}
$$

This quantity is equal to the amount of the heat stream parallel with $\mathrm{x}$-axis which passes through the plane containing $y$-axis and perpendicular to $x$-axis, and

$$
q=\int_{0}^{R} \lambda_{f}\left(-\frac{\partial F}{\partial x}\right)_{x=0} d y+\int_{R}^{b R} \lambda_{w}\left(\frac{\partial H}{\partial x}\right)_{x=0} d y \cdots(11)
$$

Because $\theta=\pi / 2$ at $\mathrm{y}$-axis and $d x=-r d \theta$, we have

$$
\begin{aligned}
& \int_{0}^{R} \lambda_{f}\left(\begin{array}{c}
\partial F \\
\partial x
\end{array}\right)_{x=0} d y \\
& =\int_{0}^{R} \lambda_{f}\left\{\frac{H_{0}}{r} \sum_{k=0}^{\infty} n A_{n}(1+\beta)\left(\frac{r}{R}\right)^{-n} \sin n \frac{\pi}{2}\right\} d r \\
& =\lambda_{f} H_{0} \sum_{k=0}^{\infty} A n(1+\beta) \sin n \frac{\pi}{2} \ldots \ldots \ldots \ldots \ldots \ldots
\end{aligned}
$$

By the similar way, we have

$$
\begin{aligned}
& \int_{R}^{b R} \lambda_{w}\left(\frac{\partial H}{\partial x}\right)_{x=0} d y \\
& =\int_{R}^{b R} \lambda_{w}\left\{H_{r}^{H_{0}} \sum_{k=0}^{\infty} n A_{n}\left\{\left(\frac{r}{k^{n}}\right)^{n}+\beta\left(\frac{r}{R}\right)^{-n}\right\} \sin n_{\vdots}^{\frac{\pi}{2}}\right\} d r \\
& =\lambda_{w} H_{0} \sum_{k=0}^{\infty} A_{n}\left\{\left(b^{n} \ldots \beta b^{n}\right)-(1-\beta)\right\} \sin n_{2}^{\pi} \cdots(13)
\end{aligned}
$$

Here, because $(1+\beta)=2 \lambda_{w} /\left(\lambda_{w}+\lambda_{f}\right)$ and $(1-\beta)=2 \lambda_{f} /\left(\lambda_{w}\right.$ $+\lambda_{f}$ ), eq. (12) and the second term of eq. (13) cancel out each other, and we have,

$$
q=\lambda_{w} H_{0} \sum_{k=0}^{\infty}(-1)^{k} A_{n}\left(b^{n-\beta} b^{-n}\right)
$$

The above equation and eq. (10) yield

$$
\frac{\lambda_{e}}{\lambda_{w}}=\frac{1}{b} \sum_{k=0}^{\infty}(-1)^{k} A_{n}\left(b^{n}-\beta b^{-n}\right)
$$

2.3 Determination of coefficients when fibers are arranged in equilateral triangles

Considering the arrangement shown in Fig. 2, the rectangle AOA' O' becomes a unit which is repeated in the whole domain. Here, planes AO' and $\mathrm{OA}^{\prime}$ the adiabatic and planes AO and O'A' are isothermal.

In the same way as the case of the arrangement at right angles, eqs. (6) and (7) are valid under the conditions of adiabatic on plane OA', isothermal on plane AO (temperature is $0^{\circ} \mathrm{C}$ ) and the continuity of temperature and heat stream on the fiber surface. Next, the temperature at an arbitrary point $\mathrm{P}$ on straight line $\mathrm{CC}^{\prime}$ perpendicular to line OO' at its center B is considered. New, points C" and C", are determined as symmetric with points $C$ and $C$ "' about $y$ axis, and $\mathrm{B}^{\prime} \mathrm{P}^{\prime}$ is put equal to $\mathrm{BP}$. The temperature at point $\mathbf{P}$ is higher by $a H_{0}$ than that at point $\mathbf{P}$ ', because the temperature at $0^{\prime}$ is higher by $a H_{0}$ than that at point 0 .

Now, defining $\overline{\mathrm{PO}^{\prime}}=\overline{\mathbf{P}^{\prime} \mathrm{O}}=\mathrm{L}(\mathrm{cm}), \angle 0^{\prime} \mathrm{OA}^{\prime}=\varphi(\mathrm{rad})$ and $\varphi-\theta=\phi(\mathrm{rad})$, we have

$$
\begin{aligned}
& \cos n \theta=\cos n(\varphi-\dot{\phi}) \\
& \quad=\cos n \varphi \cos n \phi+\sin n \varphi \sin n \phi \\
& \cos n(\pi-\varphi-\phi)=(\cos n \varphi \cos n \phi \\
& -\sin n \varphi \sin n \phi) \cos n \pi
\end{aligned}
$$

Because $n$ is odd, $\cos n \pi=-1$. This and eq (6) give the temperature difference between points $\mathrm{p}$ and $\mathrm{p}$, and on plane $\mathrm{CC}^{\prime}$

$$
a=\sum_{k=\mathbf{0}}^{\infty} 2 A_{n}\left\{\left(\frac{L}{R}\right)^{n}+\beta\left(\frac{L}{R}\right)^{-n}\right\} \cos n \varphi \cos n \phi \cdots(16)
$$


where $L=R \sqrt{a^{2}+b^{2}} /(2 \cos \phi)$.

Because adiabatic on plane AC, eq. (9) must be valid. However, when the fiber arrangement is changed and $b>a$, namely $\varphi>\pi / 4$, and points $C$ and C' are on lines AO and O'A respectively, eq. (8) holds on plane $C^{\prime} A^{\prime}$, namely isothermal condition.

The method to determine the effective heat conductivity when $\mathrm{An}$ is determined is similar to that in the right angle arrangement. But eq. (15) is valid only when $\varphi \leqq \pi / 4$. When the fiber arrangement is changed, and $b>a$, namely at $\varphi>\pi / 4$, it is better to consider the amont of heat stream across plane OO'. Namely, the amount of heat stream across plane OB, $q_{1}(\mathrm{cal} / \mathrm{sec})$, is represented by

$$
\begin{aligned}
q_{1} & =\int_{0}^{R} \lambda_{f}\left(-\frac{1}{r} \frac{\partial F}{\partial \theta}\right)_{\theta=\varphi} d r \\
& +\int_{R}^{\sqrt{\frac{a}{a_{2}+b^{2}}}}{ }^{2} \lambda_{w}\left(-\frac{1}{r} \frac{\partial H}{\partial \theta}\right)_{\theta=\varphi} d r
\end{aligned}
$$

The amount of heat stream across plane BO', $q_{2}(\mathrm{cal} / \mathrm{sec})$, is equal to the amount of the heat stream across plane $\mathrm{OB}$ ' and can be obtained by substituting $\theta=\pi-\varphi$ for $\theta=\varphi$ in eq. (17).

The following equation can be obtained similarly as in case of eqs. (12) and (13).

$$
\begin{aligned}
& \int_{0}^{R} \lambda_{f}\left(-\frac{1}{r} \frac{\partial F}{\partial \theta}\right)_{\theta=\varphi} d r=\lambda_{f} H_{0} \sum_{k=0}^{\infty} A_{n}(1+\beta) \sin n \varphi \\
& \int_{R}^{\sqrt{a} \frac{a+b \overrightarrow{b z}}{2} R} \lambda_{w}\left(-\frac{1}{r}-\frac{\partial H}{\partial \theta}\right)_{\theta=c} d r \\
& =\lambda_{w} H_{0} \sum_{k=0}^{\infty} A_{n}\left\{\left(\frac{\sqrt{a^{2}+b^{2}}}{2}\right)^{n}-\beta\left(\begin{array}{c}
\sqrt{a^{2}} b^{2} \\
2
\end{array}\right)^{-n}\right\} \\
& \times \sin n \varphi-\lambda_{w} H_{0} \sum_{k=0}^{\infty} A_{n}(1-\beta) \sin n \varphi \cdots \cdots(18)
\end{aligned}
$$

Here, similarly as in eq. (14), $q_{1}$ becomes equal to the first term at the right side of eq. (18). By substituting $\sin n(\pi-\varphi)$ for $\sin n \varphi$ in eq. (18), $q_{2}$ can be obtained. But as $\sin n(\pi-\varphi)$ is equal to $\sin n \varphi, q_{1}=q_{2}$ when $n$ is odd. So, we have

$$
\begin{aligned}
q= & \lambda_{w} H_{0} \sum_{k=0}^{\infty} 2 A_{n}\left\{\left(\frac{\sqrt{a^{2}+b^{2}}}{2}\right)^{n}-\beta\left(\frac{\sqrt{a^{2}+b^{2}}}{2}\right)^{-n}\right\} \\
& \times \sin n \varphi
\end{aligned}
$$

As this $q$ is equal to $\lambda_{e} \mathrm{bH}_{0}$ in eq. (10), we have

$$
\begin{aligned}
\lambda_{e}= & \frac{1}{b} \sum_{k=0}^{\infty} 2 A_{n}\left\{\left(\frac{\sqrt{a^{2}+b^{2}}}{2}\right)^{n}-\beta\left(\frac{\sqrt{a^{2}+b^{2}}}{2}\right)^{-n}\right\} \\
& \times \sin n \varphi
\end{aligned}
$$

where $\sqrt{a^{2}+b^{2}} / 2$ may be substituted by $a /(2 \cos \varphi)$ or $b /(2 \sin$ $\varphi)$.

\section{Determining $A_{n}$ by the least square method}

$A_{n}$ must be determined so as to fulfill the boundary conditions, isothermal for eq. (8) and adiabatic for eq. (9) in case of the right angle arrangement. In case of the equilateral triangle arrangement, the boundary conditions are eq. (16) and either eq. (8) or (9). On this basis, An were determined by the least square method.
3.1 Right angle arrangement

$f_{n}(\theta), g(\theta), f_{n}{ }^{\prime}(\theta)$ and $g^{\prime}(\theta)$ are defined as follows.

$$
\begin{aligned}
f_{n}(\theta) & =\left\{\left(\frac{a}{\cos \theta}\right)^{n}+\beta\left(\frac{\cos \theta}{a}\right)^{n}\right\} \cos n \theta \\
g(\theta) & =a
\end{aligned}
$$

where $0 \leqq \theta \leqq \tan ^{-1} b / a$.

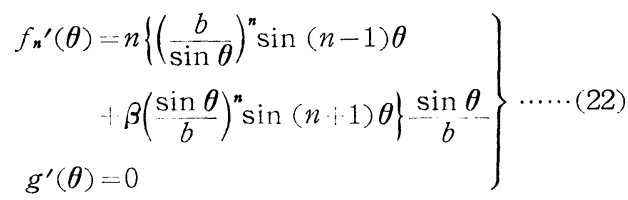

where $\tan ^{-1} b / a \leqq \theta \leqq \pi / 2$.

If the mean square value of differences between the boundary conditions and the calculated values is defined as $I$, we have from eqs. (21) and (22),

$$
\begin{aligned}
I= & \frac{1}{(a+b) R}\left[\int_{0}^{b R}\left\{g(\theta)-\sum_{k=0}^{\infty} A_{n} f_{n}(\theta)\right\}^{2} d s+\right. \\
& \left.\int_{b R}^{(a+b) R}\left\{g^{\prime}(\theta)-\sum_{k=0}^{\infty} A_{n} f_{n}^{\prime}(\theta)\right\}^{2} d s\right] \ldots . .
\end{aligned}
$$

here, the integrating variant " $S$ " is measured along the path C'BA' in Fig. 1.

To determine $A_{n}$ so as to make I minimum, the simultaneous linear equations of $A_{1}, A_{3}, A_{5}, \ldots A_{n} \ldots \ldots$ are solved by setting $\partial I / \partial A_{n}=0$.

If $Q_{n}$ and $W_{n m}$ are defined as

$$
\begin{aligned}
Q_{n}=(a+b) R & \frac{1}{(a+b R} g(\theta) f_{n}(\theta) d s \\
& \left.\int_{b R}^{(a+b) R} g^{\prime}(\theta) f_{n}^{\prime}(\theta) d s\right\} \\
W_{n m}=(a+b) R\{ & \int_{0}^{b R} f_{n}(\theta) f_{m}(\theta) d s+ \\
& \int_{b R}^{(a+b) R} f_{n^{\prime}}(\theta) f_{m^{\prime}}(\theta) d s
\end{aligned}
$$

the simultaneous equations (25) are obtained.

$$
\begin{array}{ccccc:c}
Q_{1} & W_{1,1} & W_{1,3} & \ldots \ldots \ldots \ldots \ldots & A_{1} \\
Q_{3} & W_{3,1} & W_{3,3} & \ldots \ldots \ldots \ldots \ldots & A_{3} \\
\vdots & \vdots & \ldots \ldots \ldots \ldots \ldots . & \vdots \\
Q_{n} & \vdots & \vdots & W_{n m} \ldots \ldots & A_{n} \\
\vdots & \vdots & \vdots & \vdots & \vdots
\end{array}
$$

\subsection{Equilateral triangle arrangement}

The integration along the path $\mathrm{C}^{\prime} \mathrm{CA}$ in Fig. 2 is considered when $a>b$. Similarly as the case of the right angle arrangement, $f_{n}^{\circ}(\theta)$ and $g^{\circ}(\theta)$ are defined as follows, when where $0 \leqq \theta \leqq 2 \varphi$,

$$
\begin{array}{cl}
f_{n}^{\circ}(\theta)=2\left\{\left(\frac{\sqrt{a^{2}+b^{2}}}{2 \cos \phi}\right)^{n}+\beta\left(\frac{\sqrt{a^{2}+b^{2}}}{2 \cos \phi}\right)^{-n}\right\} \\
\quad \times \cos n \varphi \cos n \phi
\end{array}
$$


$2 \varphi \leqq \theta \leqq \pi / 2$, eq. (22) can be used.

when

By defining the lengths $\overline{\mathrm{C}^{\prime} \mathrm{C}}$ and $\overline{\mathrm{CA}}$ as $l_{1}$ and $l_{2}$, we have eq. (27) in stead of eq. (23).

$$
\begin{aligned}
I= & =\frac{1}{l_{1} i l_{2}}\left[\int_{0}^{l_{1}}\left\{g^{\circ}(\theta)-\sum_{k=0}^{\infty} A_{n} f_{n}^{\circ}(\theta)\right\}^{2} d s:\right. \\
& \left.\int_{l_{1}}^{l_{1}+l_{2}}\left\{g^{\prime}(\theta) \cdots \sum_{k=0}^{\infty} A_{n} f_{n^{\prime}}(\theta)\right\}^{2} d s\right] \cdots \cdots \cdots(27)
\end{aligned}
$$

Here, similarly as the case of the right angle arrangement, $A_{n}$ can be obtained from eq. (25) when $Q_{n}$ and $W_{n m}$, are defined as:

$$
\begin{aligned}
& Q_{n}=\frac{1}{l_{1}+l_{2}}\left\{\int_{0}^{l_{1}} g^{\circ}(\theta) f^{\circ}(\theta) d s: \int_{l_{1}}^{l_{1}+l_{2}} g^{\prime}(\theta) f_{n^{\prime}}(\theta) d s\right\} \\
& W_{n m}=\frac{1}{l_{1+l}+l_{2}}\left\{\int_{0}^{l_{1}} f_{n}^{\circ}(\theta) f_{m}^{\circ}(\theta) d s+\right. \\
& \left.\quad \int_{l_{1}}^{l_{1}+l_{n}} f_{n^{\prime}}(\theta) f_{m^{\prime}}(\theta) d s\right\} \quad \cdots \cdots(28)
\end{aligned}
$$

In case of $b>a$, eq. (21) is used when $0 \leq \theta \leq 2 \varphi-\pi / 2$ and eq. (26) is used when $2 \varphi-\pi / 2 \leq \theta \leq \pi / 2$. If the integrating lengths $\overline{A^{\prime} C^{\prime}}$ and $\overline{C^{\prime} C}$ are put as $l_{0}$ and $l_{1}$, we have eq. (29) instead of eq. (27).

$$
\begin{aligned}
I= & \frac{1}{l_{0}+l_{1}}-\left\{\int_{0}^{l_{0}}\left\{g(\theta)-\sum_{k=0}^{\infty} A_{n} f_{n}(\boldsymbol{\theta})\right\}^{2} d s\right. \\
& \left.\int_{l_{0}}^{l_{0}+l_{1}}\left\{g^{\circ}(\boldsymbol{\theta})-\sum_{k=0}^{\infty} A_{n} f_{n}{ }^{\circ}(\boldsymbol{\theta})\right\}^{2} d s\right] \cdots \ldots \ldots(29)
\end{aligned}
$$

Then, defining $Q_{n}$ and $W_{n m}$ as follows, $A_{n}$ can be obtained from eq. (25), similarly to eq. (30):

$$
\begin{aligned}
& Q_{n}= \frac{1}{l_{0}+l_{1}}\left\{\int_{0}^{l_{0}} g(\boldsymbol{\theta}) f_{n}(\theta) d s+\right. \\
&\left.\int_{l_{0}}^{l_{0}+l_{1}} g^{\circ}(\boldsymbol{\theta}) f_{n} \circ(\boldsymbol{\theta}) d s\right\} \\
& W_{n m}=\frac{1}{l_{0}}\left\{l _ { 1 } \left\{\int_{0}^{l_{0}} f_{n}(\boldsymbol{\theta}) f_{m}(\boldsymbol{\theta}) d s\right.\right. \\
&\left.\quad \int_{l_{n}}^{l_{0} l_{1}} f_{n}^{\circ}(\boldsymbol{\theta}) f_{m}^{\circ}(\boldsymbol{\theta}) d s\right\}
\end{aligned}
$$

\section{Numerical calculation}

[ $\left.W_{n m}\right]$ in eq. (25) is a symmetric matrix, but not orthogonal. Even if $n \neq m, W_{n m}$ is not zero. So, eq. (25) can not be solved so easily as in the case of Fourier series. Moreover, as the integration of $Q_{n}$ and $W_{n m}$ can not be performed easily, these are obtained by numerical integration using a mini computer as follows:

In case of the right angle arrangement; $a=b$.

In case of the equilaterally triangle arrangement; $\varphi=\pi / 6$.

$$
\lambda_{f} / \lambda_{w}=0 \sim \infty .
$$

Pitch of arrangement; from the maximum filling state to $\infty$. Tables 1 and 2 show the examples of $Q_{n}, W_{n m}$ and $A_{n}$ calculated. Theoretically, simaltaneous equations having infinite unknowns must be solved. However, equations having about 20 unknowns are sufficient. As shown in the values of $\left[W_{n m}\right]$, because the diagnonal elements are only slightly larger than the other elements, the convergence by the repetition method is slow, and the accurate values can be obtained more rapidly by the elimination method.

In this report, the calculation was performed as far as $A_{n}$ and $\lambda_{e} / \lambda_{w}$ might not change over the error range of $1 / 1000$ even if the number of $A_{n}$ was increased.

This number of $A_{n}$ decreases rapidly as the fiber volume ratio decreases. When this ratio is under $60 \%$, four or five $A_{n}$ are sufficient for the calculation.

Figs. 3-6 show the examples of the temperature distributions calculated based on $A_{n}$ obtained. Figs. 7-9 also show the effective heat conductivity calculated. Figs. 7 and 8 show that in case of the most closely packed state where 0.01

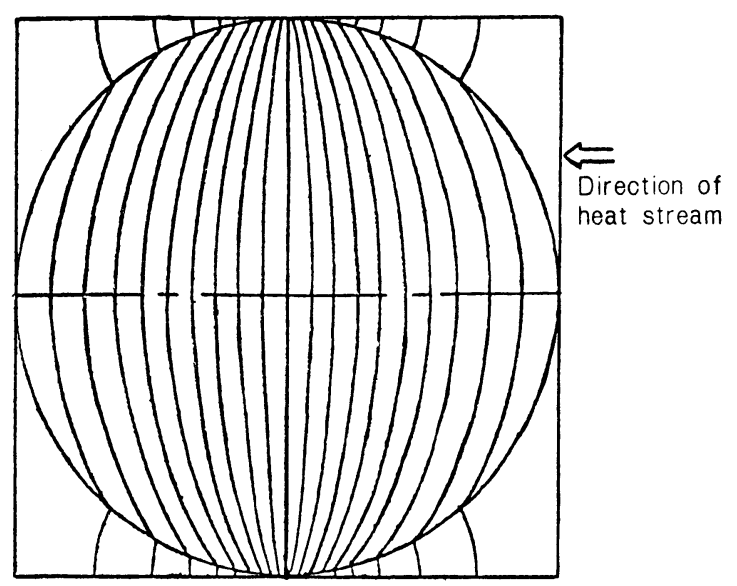

Fig. 3 Example of calculated temperature distribution (1) Right angle arrangement, most closely packed $\lambda_{f} / \lambda_{w}=0.1, \lambda_{e} / \lambda_{w}=0.16$

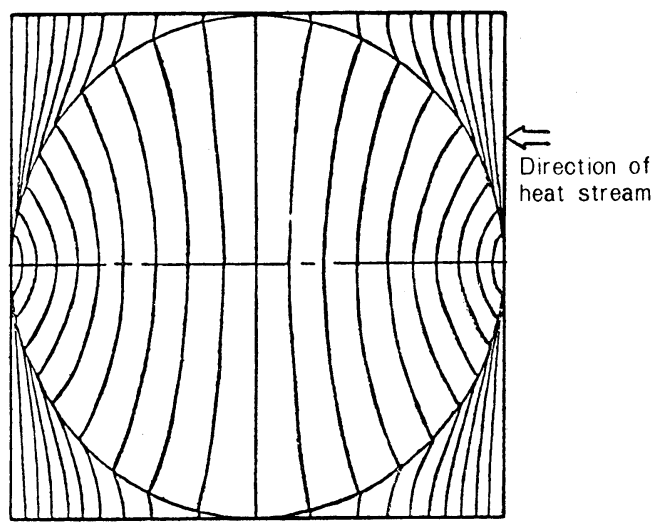

Fig. 4 Example of calculated temperature distribution (2) Right angle arrangement, most closely packed, $\lambda_{f} / \lambda_{w}=10, \lambda_{e} / \lambda_{w}=6.0$ 
$\leqq \lambda_{f} / \lambda_{w} \leqq 100$, the relations between $\lambda_{e} / \lambda_{w}$ and $\lambda_{f} / \lambda_{w}$ become as follows:

In case of the right angle arrangement;

$$
\lambda_{e} / \lambda_{w} \fallingdotseq\left(\lambda_{f} / \lambda_{w}\right)^{0.78}
$$

In case of the equilateral triangle arrangement;

$$
\lambda_{c} / \lambda_{w} \fallingdotseq\left(\lambda_{f} / \lambda_{w}\right)^{0.88}
$$

The indices in these equations $(0.78,0.88)$ are nearly equal to the fiber volume ratio in the fiber assembly in each case (0.785, 0.907).

In Fig. 9 is plotted $\lambda_{e} / \lambda_{w}$ against the fiber volume ratio $\varepsilon$. The $\lambda_{e} / \lambda_{w}$ difference between the right angle arrangement and the equilateral triangle arrangement is little when $\varepsilon<$ $60 \%$, but this difference becomes very large when $\varepsilon>70 \%$. When fibers are packed up without special consideration, it is naturally thought that the fiber arrangement becomes the equilateral triangle.

If needed, $\lambda_{e} / \lambda_{w}$ is obtained from the case $\lambda_{f} / \lambda_{w}>1$ or $\lambda_{f} / \lambda_{w}$ $<1$, because $\lambda_{e} / \lambda_{w}$ is almost reciprocal to $\lambda_{f} / \lambda_{w}$ and $\lambda_{w} / \lambda_{f}$.

\section{Comparison with reported values}

Kamata $^{[1]}$ obtained by numerical analysis the temperature distribution of the same complex in which the fibers were arranged most closely at right angles and when $\lambda_{f} / \lambda_{w}=10$, as shown in Fig. 4. Horikawa and others ${ }^{[2]}$ reported, as the experimental result by the electric current method, the temperature distribution of the complex in which the fibers were arranged most closely in the same equilateral triangle as those shown in Figs. 5 and 6 and when $\lambda_{f} / \lambda_{w} \fallingdotseq 5.6$ which is similar to that in Fig. 6. These results agree very well with those in Figs. 4 and 6.

As for the effective heat conductivity, Kamata ${ }^{[1]}$ proposed from numerical analysis the following equation when $0.1 \leqq$ $\lambda_{f} / \lambda_{w} \leqq 100$ :

$$
\lambda_{e} / \lambda_{w} \fallingdotseq\left(\lambda_{f} / \lambda_{w}\right)^{0.778}
$$

This also agrees well with eq. (30) in this report. Even

\begin{tabular}{|c|c|c|c|c|c|c|c|}
\hline \multicolumn{8}{|c|}{$a=b=1, \quad \lambda_{f} / \lambda_{w}=0.01$} \\
\hline$Q_{1}$ & \multirow{6}{*}{$\approx=$} & $W_{1,1}$ & $W_{1,3}$ & $W_{1,5}$ & $W_{1,7}$ & $\ldots \ldots \ldots \ldots$ & $A_{1}$ \\
\hline$Q_{3}$ & & $W_{3,1}$ & $W_{3,3}$ & $W_{3,5}$ & $W_{3,7}$ & & $A_{3}$ \\
\hline$Q_{5}$ & & $W_{\tilde{5}, 1}$ & $W_{5,3}$ & $W_{5,5}$ & $W_{5,7}$ & $\ldots \ldots \ldots \ldots$ & $A_{5}$ \\
\hline$Q_{7}$ & & $W_{7,1}$ & $W_{7,3}$ & $W_{7,5}$ & $W_{7,7}$ & n........ & $A_{7}$ \\
\hline$\vdots$ & & $\vdots$ & $\vdots$ & $\vdots$ & $\vdots$ & & $\vdots$ \\
\hline$\vdots$ & & $\vdots$ & $\vdots$ & $\vdots$ & $\vdots$ & & $\vdots$ \\
\hline 0.8849 & \multirow{6}{*}{$=$} & 1.713 & 0.849 & -2.200 & -2.211 & $\ldots \ldots \ldots \ldots$ & 0.6220 \\
\hline 0.1225 & & 0.849 & 4.979 & -4.195 & -25.031 & ........... & -0.1691 \\
\hline-0.6667 & & -2.200 & -4.195 & 15.731 & 17.245 & $\ldots \ldots \ldots \ldots$ & 0.0834 \\
\hline-0.0102 & & -2.211 & -25.031 & 17.245 & 190.01 & ......... & -0.0513 \\
\hline$\vdots$ & & $\vdots$ & $\vdots$ & $\vdots$ & $\vdots$ & & $\vdots$ \\
\hline$\vdots$ & & $\vdots$ & $\vdots$ & $\vdots$ & $\vdots$ & & $\vdots$ \\
\hline
\end{tabular}
when the fibers are not most closely packed, Kamata's re-

Table 1 Example of calculated values in case of the right angle arrangement

Table 2 Example of calculated values in case of the equilateral triangle arrangement

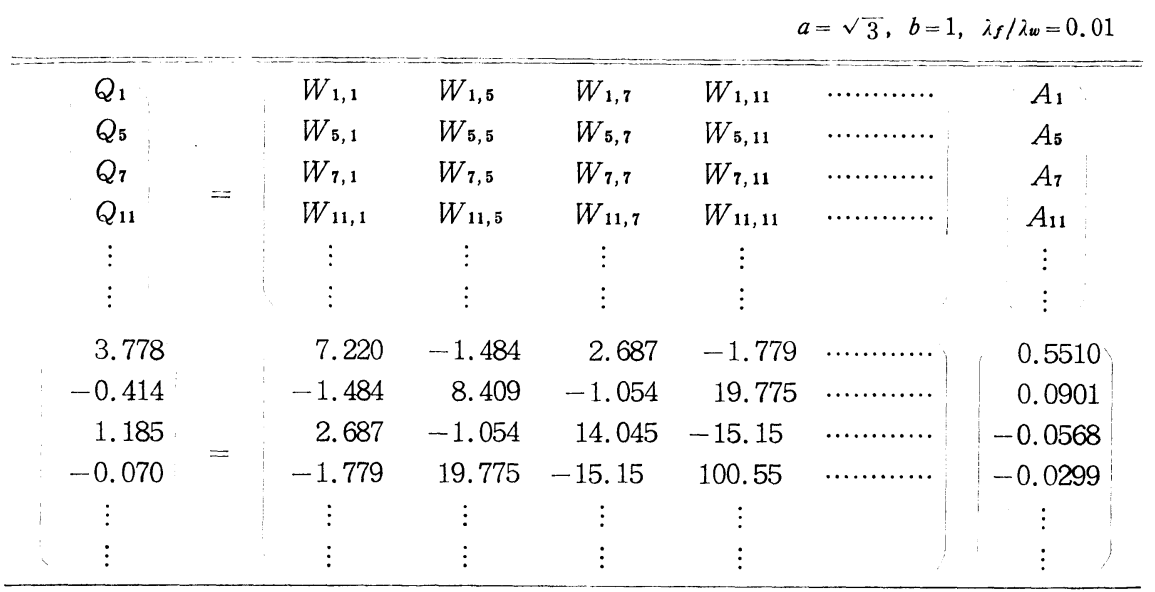

Note: In this case, the values for $n=3,9,15,21$ are zero. 


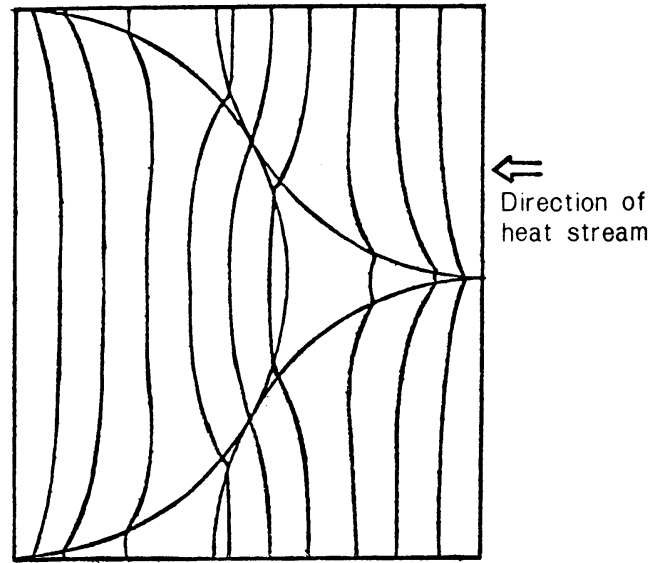

Fig. 5 Example of calculated temperature distribution (3) Equilateral triangle arrangement, most closely packed, $\lambda_{f} / \lambda_{w}=0.1, \lambda_{e} / \lambda_{w}=0.13$

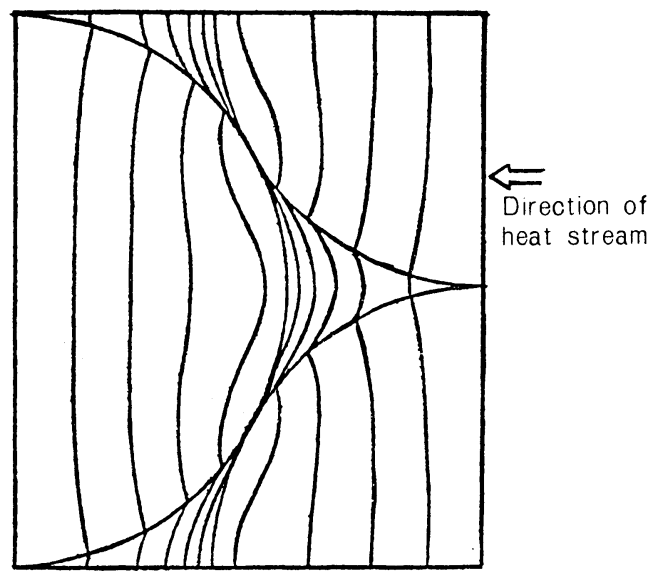

Fig. 6 Example of calculated temperature distribution (4) Equilateral triangle arrangement, most closely packed, $\lambda_{f} / \lambda_{w}=10, \lambda_{e} / \lambda_{w}=7.8$

sults agree well with the calculated values in this report. Horikawa and others ${ }^{[2]}$ also measured the effective heat conductivity by the experiment using electric current. Fig. 10 shows the comparison between these results and the calculated values in this report. They agree well, except when $\lambda_{f} / \lambda_{w}$ ratio is very small at the most closely packed arrangement.

When the fibers are most closely packed and $\lambda_{f} / \lambda_{w}$ ratio is very small, the contact part between fibers exerts much inflnence on heat transfer, and the stable result is thought very difficult to obtain.

\section{Comparison with experimental results}

Though the experiments by Horikawa and others ${ }^{[2]}$ were performed very minutely, the data very few when the fiber volume ratio was between $20 \%$ and $100 \%$. Kamata's numeri- cal analysis ${ }^{[1]}$ was limited only in the right angle arrangement and was not performed in case of the equilateral triangle arrangement which is thought to be most probable in the close packed arrangement. Therefore, the experiments under the following conditions are performed, and the results were compared between experimental and calculated.

(1) the fiber volume ratio; $20 \% \sim$ maximum

(2) $\lambda_{f} / \lambda_{w}=0$

(3) right angle arrangement, and equilateral triangle arrangement

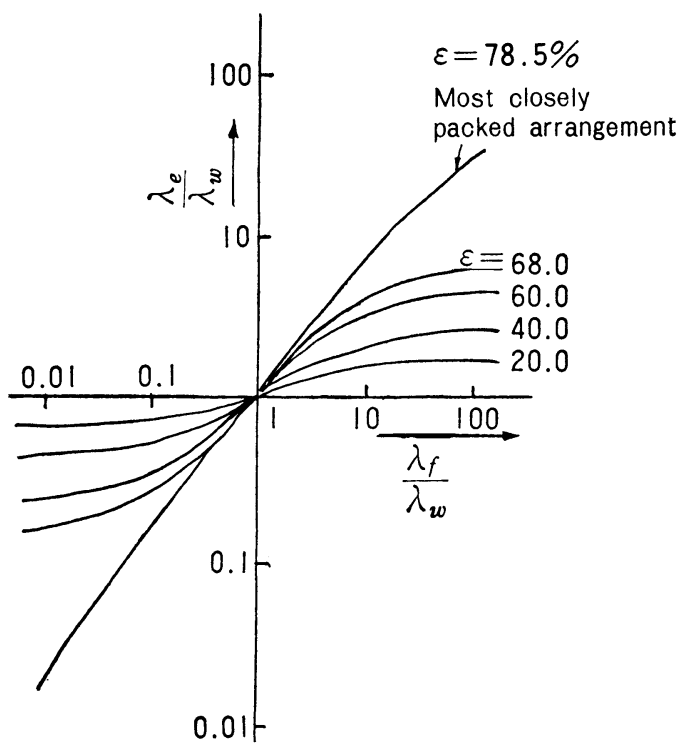

Fig. 7 Calculated effective heat conductivity; Right angle arrangement, $a=b$

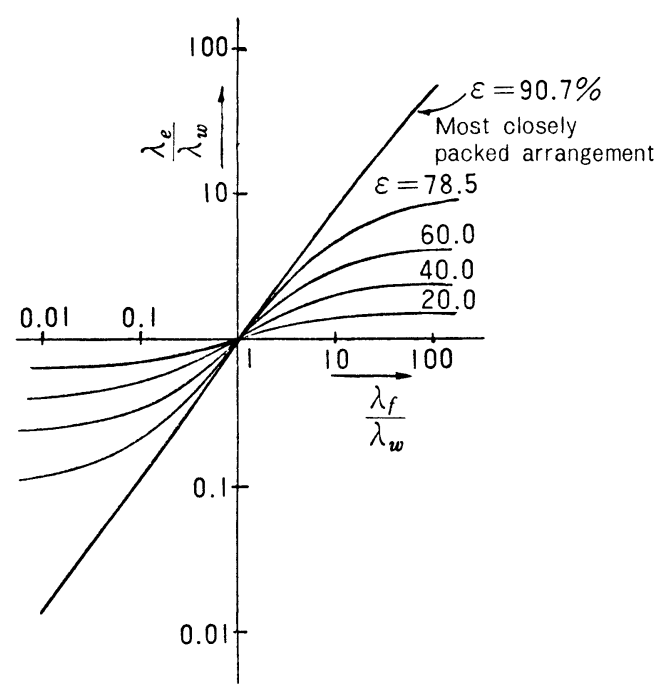

Fig. 8 Calculated effective heat conductivity; Equilateral triangle arrangement, $\varphi=\pi / 6$ 


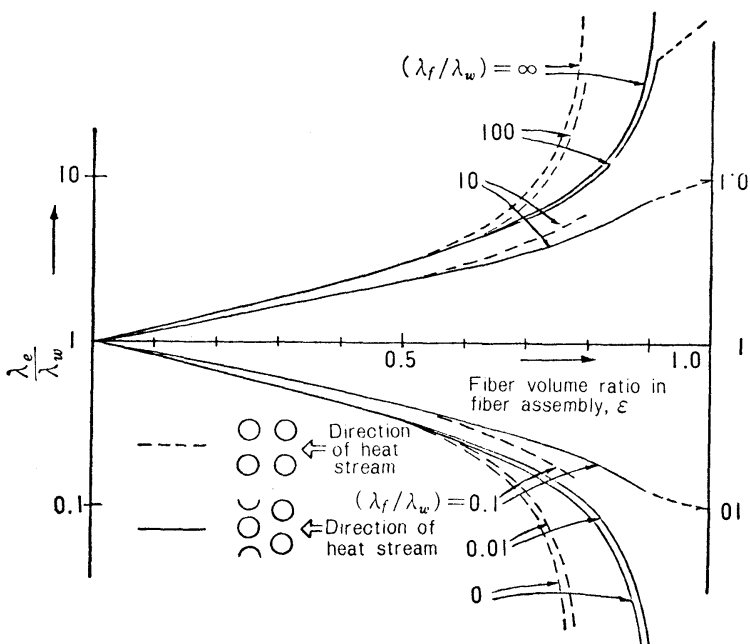

Fig. 9 Calculated effective heat conductivity

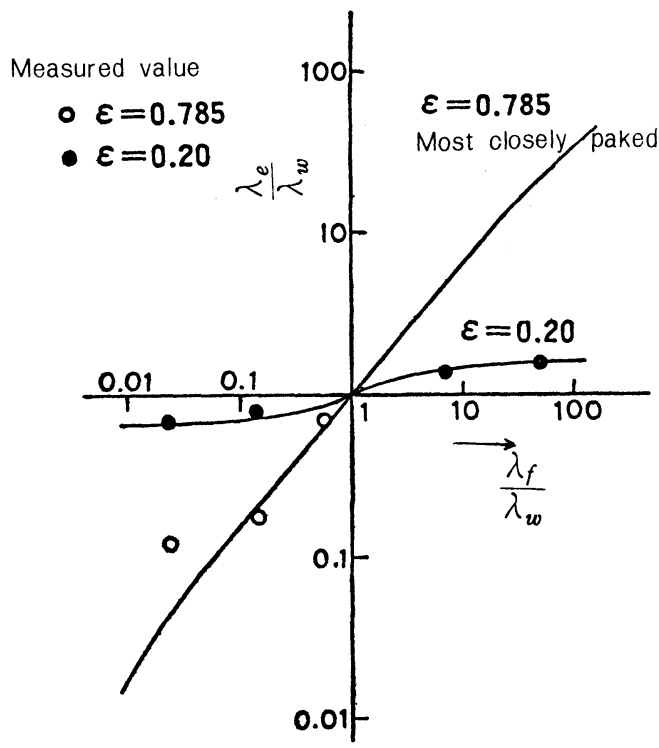

Fig. 10 Comparison between calculated and measured effective heat conductivity right angle arrangement

\subsection{Experimental method}

The measurement is based on checking the electric resistance similarly as Horikawa's experiment ${ }^{[2]}$. The material used is the paper made of activated carbon fibers and wooden pulp by Toyobo Co., the properties of which is shown in Table 3. The paper is cut off as shown in Figs. 11 14, and the electric resistances between the broken lines are measured by a digital-volt-ohm meter made by Takeda Riken. In each pattern, value $a$ is varied from 0.1 to $4.7 \mathrm{~cm}$.

To avoid the error based on the paper unevenness, the electric resistances are measured before and after the circles are cut off in the paper.

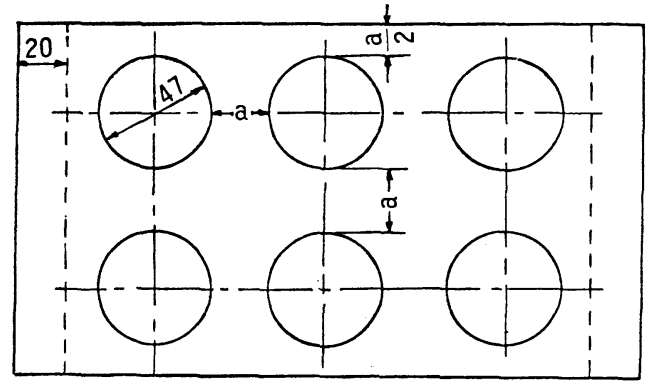

Fig. 11 Sample A for measuring electric resistance (unit; mm)

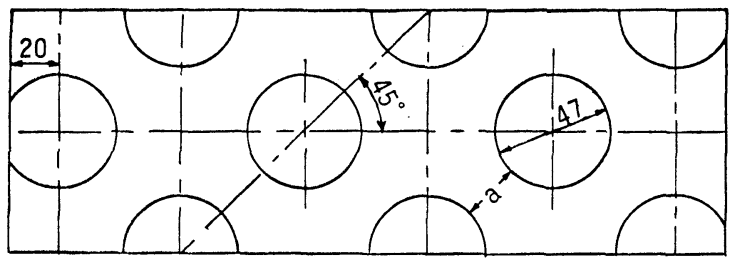

Fig. 12 Sample B for measuring electric resistance (unit; mm)

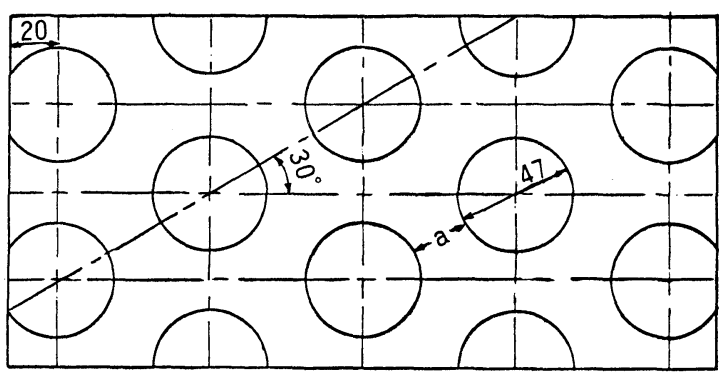

Fig. 13 Sample C for measuring electric resistance (unit; $\mathrm{mm}$ )

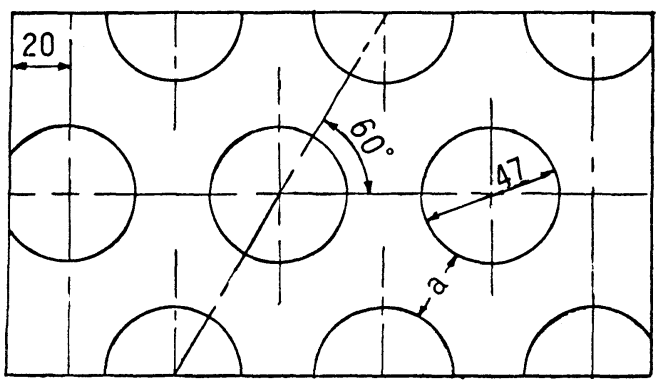

Fig. 14 Sample D for measuring electric resistance (unit; mm)

\subsection{Experimental results and discussion}

Figs. 15 and 16 show the experimental results. Pattern A corresponds to the entire right angle arrangement, and its results almost agree with the calculated values shown by the solid line. Pattern B corresponds to the equilateral triangle arrangement, and at the same time is obtained by rotating pattern A by $45^{\circ}$. The results are also shown in Fig. 15 and 
Table 3 Properties of carbon-fiber paper used

$\begin{array}{ll}\text { Carbon fiber: Wooden pulp (mass ratio) } & 3: 7 \\ \text { Mean thickness }(\mathrm{cm}) & 1.6 \times 10^{-2} \\ \text { Mean mass }\left(\mathrm{g} / \mathrm{cm}^{2}\right) & 5.6 \times 10^{-3} \\ \text { Mean electric resistance* }(\Omega) & 1.6 \times 10^{-3}\end{array}$

*: Resistance of sheet whose length and width are $10 \mathrm{~cm}$.

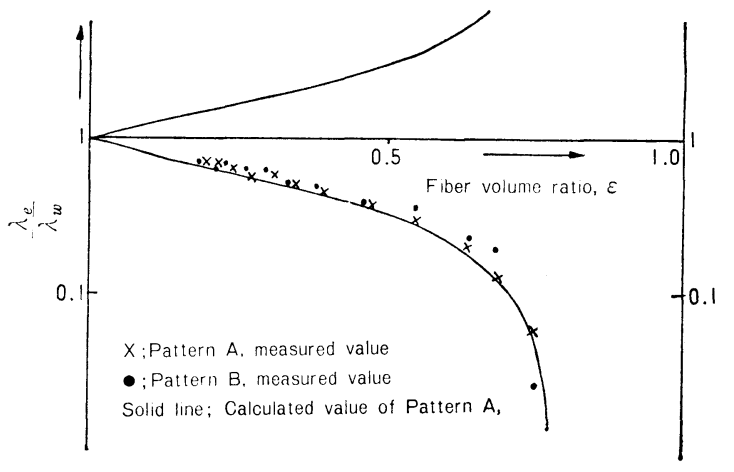

Fig. 15 Comparison between calculated and measured effective heat conductivity; $\lambda_{f}=0$, pattern $\mathrm{A}$ and $\mathrm{B}$

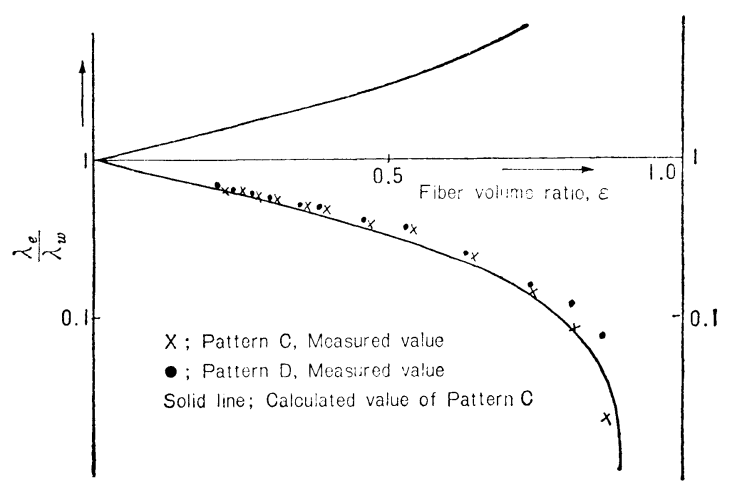

Fig. 16 Comparison between calculated and measured effective heat conductivity; $\lambda_{f}=0$, pattern $\mathrm{C}$ and $\mathrm{D}$

are the same as those of Pattern A. Thus, when the type of the arrangement and the fiber volume ratio are equal, the effective heat conductivity is thought to be almost the same, even if the direction of heat stream is changed.

Pattern C shown in Fig. 16 corresponds to the case of $\varphi=$ $\pi / 6$, and the solid line shows the calculated values in this case. Pattern $\mathrm{D}$ is obtained by rotating pattern $\mathrm{C}$ by $30^{\circ}$. Both these experimental results agree well with the calculated values. This shows that the theories in this report are right, and that the difference based on the change of heat-stream direction is little.

\section{Conclusion}

The theoretical analysis on the heat conductivity of the complex which consists of fibers and the medium existing among fibers is performed, and the results obtained are compared with both reported and experimental values. The results obtained are as follows,

(1) The equations showing the temperature distribution in the complex,

in medium; $\sum_{k=0}^{\infty} A_{n}\left\{\left(\frac{r}{R}\right)^{n}+\beta\left(\frac{r}{R}\right)^{-n}\right\} \cos n \theta$

in fiber; $\quad \sum_{k=0}^{\infty} A_{n}(1+\beta)\left(\begin{array}{c}r \\ \mathrm{R}\end{array}\right)^{n} \cos n \theta$

Where $A_{n}$ is the coefficient to satisfy the boundary conditions, and is determined by the least square method.

(2) The effective heat conductivity of the complex is, in case of the right angle arrangement;

$$
\frac{\lambda_{e}}{\lambda_{w}}=\frac{1}{b} \sum_{k=0}^{\infty}(-1)^{k} A_{n}\left(b^{n}-\beta b^{-n}\right)
$$

in case of the equilateral triangle arrangement; either the above equation or

$$
\frac{\lambda_{e}}{\lambda_{w}}=\frac{1}{b} \sum_{k=0}^{\infty} 2 A_{n}\left\{\left(\frac{\sqrt{a^{2}+b^{2}}}{2}\right)^{n}-\beta\left(\frac{\sqrt{a^{2}+b^{2}}}{2}\right)^{-n}\right\} \sin n \zeta
$$

The calculated values by these theoretical equations agree well with both reported ${ }^{[1][2]}$ and experimental values.

(3) When the fibers are packed most closely, $\lambda_{e}$ is in case of the right angle arrangement and $\left(0.01 \leqq \lambda_{f} / \lambda_{w} \leqq 100\right)$;

$$
\lambda_{e} / \lambda_{w} \fallingdotseq\left(\lambda_{f} / \lambda_{w}\right)^{0.78}
$$

in case of the equilateral triangle arrangement $(\varphi=\pi / 6)$ and $\left.0.01 \leqq \lambda_{f} / \lambda_{w} \leqq 100\right)$;

$$
\lambda_{e} / \lambda_{w} \fallingdotseq\left(\lambda_{f} / \lambda_{w}\right)^{0.88}
$$

\section{References}

[1] K. Kamata; J. Soc. Fib. Sci. Tech. Japan, 31, T317(1975)

[2] A. Horikawa, Y. Matsuda; J. Text. Mach. Soc. Japan, 27, T84 (1974)

[3] Y. Kodaira; Butsuri Sugaku Vol. 2, p. 510, 1948, Iwanami (Tokyo) 